\title{
GALACTIC LINE EMISSION FROM 1-10 keV
}

\author{
GARY STEIGMAN and JOSEPH SILK \\ Institute of Theoretical Astronomy, Cambridge, England
}

\begin{abstract}
Calculations are given of $\mathrm{K}$-series $\mathrm{X}$-rays produced by interaction of both low energy cosmic rays and diffuse $\mathrm{X}$-rays above $1 \mathrm{keV}$ with heavier ions present in HI regions. We further consider electron capture to excited states by cosmic ray nuclei of heavy elements, followed by cascades down to the ground state. It is found that the electron capture process may yield appreciable line intensities in the $1-10 \mathrm{keV}$ range in the galactic plane.
\end{abstract}

Hayakawa [1] originally suggested that low-energy cosmic rays (in the range 1-100 $\mathrm{MeV}$ ) may be an important heat source in the interstellar medium. Cosmic ray heating has been extensively studied by Hayakawa et al. [2] and more recently by Pikel'ner [3], Balasubrahmanyan et al. [4], and Spitzer and Tomasko [5], who find substantial agreement with the observed properties of the interstellar medium. However, this is at best an indirect argument for the presence of sub-cosmic rays. Indeed the observed diffuse soft X-ray flux may be of comparable significance as a heating mechanism [6]. In order to distinguish between heating by sub-cosmic rays and other possible mechanisms, it is clearly of great importance to attempt to observe low-energy cosmic rays. Direct observations at low energies are unreliable because of the substantial degree of solar modulation [7].

A more promising approach is to investigate the interactions of low-energy cosmic rays with HI regions. Observations yield an upper limit on the heating due to low energy cosmic rays [8], in the form of an integral over the cosmic ray spectrum. Greenberg [9] has discussed the radio emission lines produced by electron cascades following recombination to highly excited states. Proton inner-bremsstrahlung radiation by low energy cosmic rays interacting with the interstellar medium has been discussed by Hayakawa and Matsuoka [10], and more recently by Boldt and Serlemitsos [11]. A power-law spectrum is produced, of energy spectral index equal to that of the non-relativistic proton spectrum at photon energies above about $20 \mathrm{keV}$. However, the flux falls some two orders of magnitude or more below the observed diffuse background at $1 \mathrm{keV}$. Note that this result depends only on the heat input assumed for the low-energy cosmic rays.

The fluxes of characteristic X-ray lines from K-series transitions following K-shell ionizations by fast protons in the direction of the galactic centre have previously been discussed by Gould and Burbidge [12] and Hayakawa and Matsuoka [10]. In this paper, we extend their work to include calculations of the $\mathrm{K}$-series $\mathrm{X}$-rays produced by interaction of both low-energy cosmic ray protons and diffuse X-rays above $1 \mathrm{keV}$ with heavier ions present in $\mathrm{HI}$ regions, and of cosmic ray nuclei with atomic hydrogen. The processes that we consider are collisional ionization of $\mathrm{K}$-electrons of interstellar atoms by cosmic nuclei and subsequent $\mathrm{K}$-transitions; photo-ionizations of $\mathrm{K}$-shell electrons by diffuse $\mathrm{X}$-rays; and charge exchanges involving electron capture to 
excited states by cosmic ray nuclei of heavy elements followed by cascades down to the ground state.

The observed X-ray background is isotropic over 4-40 keV to within $10 \%$ [13], and is thought to be of extragalactic origin. The power-law spectrum is suggestive of a non-thermal mechanism, and several mechanisms have been proposed which produce continuous spectra [10]. We shall assume that the isotropic background flux is continuous for purposes of comparison with our results.

The line fluxes that we calculate are correlated with the distribution in the galaxy of neutral hydrogen, and so are most readily detectable along lines of sight lying within the galactic plane. Our results are presented in Tables I and II.

\section{TABLE I}

K-transitions following photo- and collisional ionizations

\begin{tabular}{llll} 
Element & $E(\mathrm{keV})[26]$ & $J_{\text {photo }}^{Z}\left(\mathrm{~cm}^{-2} \mathrm{sec}^{-1} \mathrm{st}^{-1}\right)$ & $J_{\text {Coll }}^{Z}\left(\mathrm{~cm}^{-2} \mathrm{sec}^{-1} \mathrm{st}^{-1}\right)$ \\
\hline $\mathrm{Na}$ & 1.1 & $6.0 \times 10^{-5}$ & $1.1 \times 10^{-5}$ \\
$\mathrm{Mg}$ & 1.3 & $2.4 \times 10^{-3}$ & $4.0 \times 10^{-4}$ \\
$\mathrm{Al}$ & $1.5,1.6$ & $2.1 \times 10^{-4}$ & $3.2 \times 10^{-5}$ \\
$\mathrm{Si}$ & $1.7,1.8$ & $4.4 \times 10^{-3}$ & $6.1 \times 10^{-4}$ \\
$\mathrm{~S}$ & $2.3,2.5$ & $5.2 \times 10^{-3}$ & $5.0 \times 10^{-4}$ \\
$\mathrm{Ar}$ & $3.0,3.2$ & $1.2 \times 10^{-4}$ & $1.1 \times 10^{-5}$ \\
$\mathrm{Ca}$ & $3.7,4.0$ & $4.5 \times 10^{-4}$ & $2.8 \times 10^{-5}$ \\
$\mathrm{Fe}$ & $6.4,7.1$ & $1.8 \times 10^{-3}$ & $3.6 \times 10^{-5}$
\end{tabular}

$E=$ Energy of $\mathrm{K} X$-ray

$J_{\text {photo }}^{Z}=$ Flux of K X-rays emitted after photo-ionizations

$J_{\text {Coll }}^{z}=$ Flux of X-rays emitted after collisional ionizations

TABLE II

$2 p-1 s$ and $3 p-1 s$ transitions following electron capture by cosmic ray nuclei

\begin{tabular}{lllll}
\hline Element & $E \alpha(\mathrm{keV})$ & $J_{\alpha}^{Z}\left(\mathrm{~cm}^{-2} \mathrm{sec}^{-1} \mathrm{st}^{-1}\right)$ & $E \beta(\mathrm{keV})$ & $J_{\beta}^{Z}\left(\mathrm{~cm}^{-2} \mathrm{sec}^{-1} \mathrm{st}^{-1}\right)$ \\
\hline $\mathrm{Ne}$ & 1.02 & 0.20 & 1.21 & 0.06 \\
$\mathrm{Na}$ & 1.23 & 0.32 & 1.46 & 0.01 \\
$\mathrm{Mg}$ & 1.47 & 0.65 & 1.74 & 0.20 \\
$\mathrm{Al}$ & 1.72 & 0.09 & 2.04 & 0.02 \\
$\mathrm{Si}$ & 2.00 & 1.12 & 2.37 & 0.31 \\
$\mathrm{P}-\mathrm{K}$ & $2.29-3.68$ & 0.41 & $2.72-4.37$ & 0.07 \\
$\mathrm{Ca}-\mathrm{Cr}$ & $4.08-5.88$ & 0.43 & $4.84-6.97$ & 0.08 \\
$\mathrm{Mn}-\mathrm{Ni}$ & $6.38-8.00$ & 0.69 & $7.56-9.49$ & 0.13 \\
\hline
\end{tabular}

$E_{\alpha}=$ Energy of $2 p-1 s$ transition

$E_{\beta}=$ Energy of $3 p-1 s$ transition

Note: For $1 \leqslant \mathrm{E} \leqslant 2(\mathrm{keV}) \frac{J \alpha+J \beta}{J \gamma}=0.31$

$J_{\alpha}^{Z}=$ Flux of X-rays with energy $E_{\alpha}$

For $2 \leqslant E \leqslant 10(\mathrm{keV}) \frac{J \alpha+J \beta}{J \gamma}=0.37$,

$J_{\beta}^{Z}=$ Flux of X-rays with energy $E_{\beta}$ where $J \gamma$ is the diffuse X-ray flux [19] in the appropriate energy interval. 
The low-energy cosmic ray flux is assumed to be mono-energetic, at $2 \mathrm{MeV}$ per nucleon. A similar assumption is made by Spitzer and Tomasko [5] and Field et al. [8] in their discussions of the heating of the interstellar medium. In the following, we describe the details of our calculations and we also mention the effects of taking different forms for the low-energy cosmic ray spectrum.

Photo-ionization. An X-ray from the diffuse background ( $E \gtrsim 1 \mathrm{keV})$ may knock a $\mathrm{K}$-shell electron out of an atom whose nuclear charge is $Z$. The cross-section for such $\mathrm{K}$-shell photo-ionizations, valid near threshold, is [14, 15]

$$
\sigma_{z}(E)=4.23 \times 10^{-23} Z_{K}^{Z^{3}}\left\{1-4.40 \times 10^{-3} \frac{Z_{K}^{2}}{E}\right\} \mathrm{cm}^{2} .
$$

The X-ray energy $E$ is in $\mathrm{keV}$. The effective charge $Z_{K}=Z-0.3$ corrects for inner screening. The probability $W_{K}^{Z}$, that the $K$-shell vacancy will be filled via $X$-ray emission (rather than by an Auger transition) is given by [16]

$$
W_{K}^{Z}=\left(1+\frac{A}{Z^{4}}\right)^{-1} ; A=\left\{\begin{array}{l}
1.19 \times 10^{6}, 10 \leqslant Z \leqslant 18 \\
1.27 \times 10^{6}, \quad Z>18
\end{array} .\right.
$$

The flux of line $X$-rays from atom $Z$ may be expressed as

$$
J_{\text {photo }}^{\mathrm{Z}}=\left\langle N_{\mathrm{H}} R\right\rangle\left(\frac{N_{\mathrm{Z}}}{N_{\mathrm{H}}}\right) W_{K}^{\mathrm{Z}} \int_{I_{K}^{\mathrm{Z}}}^{\infty} \sigma_{z}(E) j_{\gamma}(E) \mathrm{d} E \mathrm{~cm}^{-2} \mathrm{sec}^{-1} \mathrm{st}^{-1} .
$$

The number of $\mathrm{H}$ atoms along a line of sight $\left\langle N_{\mathrm{H}} R\right\rangle$ is taken to be $3 \times 10^{22} \mathrm{~cm}^{-2}$ in the direction of the galactic centre, for X-rays above $2 \mathrm{keV}$. We have neglected absorption of $\mathrm{X}$-rays at these energies. In the range $1-2 \mathrm{keV}$, we have corrected for interstellar absorption using the results of Bell and Kingston [18] to provide an effective value of $\left\langle N_{\mathrm{H}} R\right\rangle$ corresponding to optical depth unity. For directions away from the galactic centre the number of $\mathrm{H}$ atoms along a line of sight $\left\langle N_{\mathrm{H}} R\right\rangle$ is less than $3 \times 10^{22} \mathrm{~cm}^{-2}$ and the fluxes are correspondingly reduced. $I_{K}^{Z}$ is the experimental $\mathrm{K}$-shell ionization potential [27]. Below $10 \mathrm{keV}$, the diffuse X-ray photon flux may be fitted by a power-law spectrum [19].

$$
j_{\gamma}(E)=12.4 E^{-\alpha}\left(\mathrm{cm}^{-2} \mathrm{sec}^{-1} \mathrm{keV}^{-1} \mathrm{st}^{-1}\right) ; \quad \alpha=1.7 \pm 0.2 .
$$

The line X-ray fluxes have been evaluated for the most abundant atoms whose $\mathrm{K}$ $\mathrm{X}$-rays lie in the range from $1-10 \mathrm{keV}$. The results appear in Table I.

Collisional ionization. The flux of X-ray photons from atoms of charge $Z$ whose $\mathrm{K}$-electrons have been knocked out in collisions with low energy cosmic ray protons is given by

$$
J^{\mathrm{Z}}=\left\langle N_{\mathrm{H}} R\right\rangle\left(\frac{N_{\mathrm{Z}}}{N_{\mathrm{H}}}\right) W_{K}^{\mathrm{Z}} \int j(E) \sigma_{\mathrm{Z}}(E) \mathrm{d} E
$$


For the low-energy cosmic ray flux we shall adopt the $2 \mathrm{MeV}$ flux employed by Field et al [8], i.e. $4 \pi j(E)=24 \delta(E-2)$. The cross-section for K-shell ionization may be written as $[20,21]$

$$
\sigma_{Z}(E)=\frac{S(\eta)}{Z_{K}^{4}} ; \quad \eta=\frac{40}{Z_{K}^{2}} E(\mathrm{MeV}) .
$$

For $\eta \lesssim 10^{-1}, S(\eta) \sim \eta^{4}$. For $\eta \gg 1, S(\eta) \sim \eta^{-1} \ln \eta$. For intermediate values of $\eta$, $S(\eta)$ is given graphically by Merzbacher and Lewis [20]. Note that the maximum value of $S(\eta)$ occurs at $\eta \sim 1$, and is $\sim 4.5 \times 10^{16} \mathrm{~cm}^{2}$.

If the projectile is a bare nucleus of charge $Z^{\prime}$, (instead of a proton) the flux of $\mathrm{K}$ $\mathrm{X}$-rays is given by

$$
J_{Z^{\prime}}^{Z}=\left(\frac{N_{Z^{\prime}}}{N_{\mathrm{H}}}\right)_{\mathrm{CR}}\left(Z^{\prime}\right)^{2} J^{z}
$$

The quantity $\left(N_{\mathrm{Z}} / N_{\mathrm{H}}\right)_{\mathrm{CR}}$ is the abundance of nuclei with charge $Z$ relative to protons in the $2 \mathrm{MeV}$ cosmic ray flux.

The total X-ray flux caused by all cosmic ray nuclei colliding with atoms $Z$ is

$$
J_{\text {Coll }}^{\mathrm{Z}}=\sum_{Z^{\prime}}\left(\begin{array}{l}
N_{Z^{\prime}} \\
N_{\mathrm{H}}
\end{array}\right)_{\mathrm{CR}}\left(Z^{\prime}\right)^{2} J^{z} .
$$

We estimate the relative abundances of cosmic ray nuclei at $2 \mathrm{MeV}$ from the results of Comstock et al. [22], and obtain $\sum\left(N_{Z}^{\prime} / N_{\mathrm{H}}\right)_{\mathrm{CR}}\left(Z^{\prime}\right)^{2}=2.65$.

$J_{\text {Coll }}^{Z} \equiv 2.65 J^{Z}$ has been evaluated for the same elements as before. The resulting line intensities are given in Table I.

Electron capture. In an encounter with a hydrogen atom, a low-energy cosmic ray nucleus may capture the electron by the charge exchange process

$$
\mathrm{H}(1 \mathrm{~s})+A^{\mathrm{Z}(+)} \rightarrow A^{(\mathrm{Z}-1)(+)}(n l)+\mathrm{H}^{+} .
$$

The electron thus captured in an excited state, will cascade down to the ground state. If, in this process, the electron finds itself in the $2 p$ state, it will emit the analog of the Ly- $\alpha$ line and jump down to the ground state. The energy of the line thus emitted is

$$
E_{\alpha}^{Z}=1.02\left(\begin{array}{c}
Z \\
10
\end{array}\right)^{2} \mathrm{keV},
$$

where $Z$ is the charge of the cosmic ray nucleus.

Similarly, if the electron finds itself in the $3 p$ state, it will emit the analog of the Ly- $\beta$ line and jump down to the ground state, $88 \%$ of the time. The other $12 \%$ of the time it will end up in the metastable $2 s$ state. The energy of this line is

$$
E_{\beta}^{Z}=1.21\left(\frac{Z}{10}\right)^{2} \mathrm{keV} .
$$


The cross-section for Ly- $\alpha$ emission may be expressed as

$$
\sigma_{\alpha}^{Z}=\sum_{l=0}^{\infty} \sum_{n=l+1}^{\infty} f_{n l}^{\alpha} \sigma_{1 s-n l}^{Z} .
$$

$f_{n l}$ is the probability that an electron, initially in the state $n l$, will ultimately make the $2 p-1 s$ transition. $\sigma_{1 s-n l}^{Z}$ is the cross-section for a nucleus of charge $Z$ to capture an electron from the ground state of hydrogen into the $n l$ state. The corresponding cross section for Ly- $\beta$ emission is

$$
\sigma_{\beta}^{Z}=\sum_{l=0}^{\infty} \sum_{n=l+1}^{\infty} f_{n l}^{\beta} \sigma_{1 s-n l}^{Z} .
$$

$f_{n l}^{\beta}$ is the probability an electron captured into the state $n l$ will ultimately make the $3 p-1 s$ transition.

The probabilities $f_{n l}^{\alpha}, f_{n l}^{\beta}$ have been evaluated by Bethe and Salpeter [23]. Bates and Dalgarno [24] have evaluated the electron cros-ssections for all states through $n=4$, $l=3$. Hiskes [25] has given expressions for $\sigma_{1 s-n l}$ for all states through $n=15, l=14$.

The flux of line emission from electron capture is

$$
\begin{aligned}
& J_{\alpha}^{\mathrm{Z}}=\left\langle N_{\mathrm{H}} R\right\rangle\left(\frac{N_{\mathrm{Z}}}{N_{\mathrm{H}}}\right)_{\mathrm{CR}} \int j(E) \sigma_{\alpha}^{\mathrm{Z}}(E) \mathrm{d} E, \\
& J_{\beta}^{\mathrm{Z}}=\left\langle N_{\mathrm{H}} R\right\rangle\left(\frac{N_{\mathrm{Z}}}{N_{\mathrm{H}}}\right)_{\mathrm{CR}} \int j(E) \sigma_{\beta}^{\mathrm{Z}}(E) \mathrm{d} E .
\end{aligned}
$$

As previously, we employ the flux $4 \pi j(E)=24 \delta(E-2)$ and take $\left(N_{Z} / N_{\mathrm{H}}\right)_{\mathrm{CR}}$ from Comstock et al. [22]. The heavy nuclei are assumed to be present at an energy of $2 \mathrm{MeV} /$ nucleon. As before, we take $\left\langle N_{\mathrm{H}} R\right\rangle=3 \times 10^{22} \mathrm{~cm}^{-2}$.

For $E=2 \mathrm{MeV}$ and $10 \leqslant Z \leqslant 26$, the cross-sections $\sigma_{\alpha}^{Z}$ and $\sigma_{\beta}^{Z}$ were estimated from (11) and (12) by including all contributions through the $4 f$ state. The resulting crosssections are therefore lower limits to the exact cross-sections. They are most accurate for low $Z$. For $Z \gtrsim 20$ they may be too small by an order of magnitude. The results appear in Table II. The lines below $2 \mathrm{keV}$ have been corrected for interstellar absorption, as previously described.

Although none of the K-lines due to collisional or photo-ionizations should be observable, it appears from Table II that it would be feasible to look for the $2 p-1 s$ transitions following electron capture by low energy cosmic ray nuclei. In our calculation of the line fluxes for the $2 p-1 s$ and $3 p-1 s$ transitions we have used cross-sections which in fact are lower limits to the exact cross-sections. Measurements of line intensities (or null results) would therefore yield upper limits to the flux of cosmic ray nuclei with $10 \leqslant Z \leqslant 28$ at $2 \mathrm{MeV}$ per nucleon. It should be noted that these lines will be Doppler broadened. Since $\Delta E / E \simeq 0.13$, the lines with $E \gtrsim 2 \mathrm{keV}$ will begin to overlap, making them more difficult to resolve.

Throughout the discussion of X-ray lines produced by low-energy cosmic rays we have used the $2 \mathrm{MeV}$ flux employed by Field et al. [8]. It is of interest to know how these results are modified if a more realistic spectrum (say, an extrapolation of the 
demodulated spectrum of high energy cosmic rays) is used. A detailed investigation of this is in progress but some general remarks may be made. In the case of collisional ionization, the reduced cross-section $S(\eta)$ (see Equation (7)) is rather slowly varying in the neighbourhood of its maximum $(0.1 \lesssim \eta \lesssim 10)$. A rough estimate of $J_{\text {Coll }}^{Z}$ may be obtained as

$$
J_{\text {Coll }}^{\mathrm{Z}} \approx 2.65\left\langle N_{\mathrm{H}} R\right\rangle\left(\frac{N_{\mathrm{Z}}}{N_{\mathrm{H}}}\right) W_{K}^{\mathrm{Z}}\left\langle\sigma_{\mathrm{Z}}\right\rangle \int_{\eta=0.1}^{\eta=10} j(E) \mathrm{d} E ; \quad \eta=\frac{40 E}{Z^{2}} .
$$

Such estimates of $J_{\text {Coll }}^{Z}$ indicate order of magnitude agreement with the previous results.

For energies $\gg 2 \mathrm{MeV}$, the cross-sections $\sigma_{\alpha}^{Z}, \sigma_{\beta}^{Z}$ are very small $\left(\sigma \sim E^{-6}\right)$. Thus, most of the contributions to $\sigma_{\alpha}^{Z}$ and $\sigma_{\beta}^{Z}$ come in an energy interval around or less than $2 \mathrm{MeV}$. However, even if the cosmic ray spectrum peaks at a somewhat higher energy than $2 \mathrm{MeV}$, we would still predict an appreciable flux at $2 \mathrm{MeV}$. Ionization losses tend to produce a spectrum of positive slope at low energies, and the spectrum would be unlikely to peak much above $15 \mathrm{MeV}$, otherwise the cosmic ray heating would be drastically reduced. Although this gives an effective reduction in the flux at $2 \mathrm{MeV}$, the integration over a cosmic-ray spectrum tends to enhance the line intensities. Moreover, even a small flux below $2 \mathrm{MeV}$ makes an important contribution to the lines because of the steep dependence on energy of the cross-sections for $2 p-1 s$ and $3 p-1 s$ transitions. The net result appears to be that the line fluxes are not significantly reduced by inclusion of a more realistic spectrum.

We would like to thank Drs. G. B. Field, M. J. Rees and M. W. Werner for helpful comments on this work.

\section{References}

[1] Hayakawa, S.: 1960, Publ. Astron. Soc. Japan, 12, 110.

[2] Hayakawa, S., Nishimura, S., and Takayanagi, K.: 1961, Publ. Astron. Soc. Japan 13.

[3] Pikel'ner, S. B.: 1968, Soviet Astron.-AJ 11, 737.

[4] Balasubrahmanyan, V. K., Boldt, E., Palmeira, R. A. R., and Sandri, G.: 1968, Can. J. Phys. 46, 5633.

[5] Spitzer, L., and Tomasko, M. G.: 1968, Astrophys. J. 152, 971.

[6] Silk, J. and Werner, M. W.: 1969, Astrophys. J. 158, 185.

[7] Gloeckler, G. and Jokipii, J. R.: 1967, Astrophys. J. 148, 241.

[8] Field, G. B., Goldsmith, D. W., and Habing, H. J.: 1969, Astrophys. J. 155, L149. The flux of $2 \mathrm{MeV}$ cosmic rays used by these authors is about half that assumed in Reference [5], as heavy nuclei were included. This increases the heat input of a given flux of low-energy cosmic rays at $2 \mathrm{MeV} /$ nucleon by a factor $\Sigma_{Z}\left(N_{Z}^{\prime} / N_{H}\right) \mathrm{CR}\left(Z^{\prime}\right)^{2}$.

[9] Greenberg, D. W.: 1969, Astrophys. J. 155, 451.

[10] Hayakawa S. and Matsuoka M.: 1964, Supp. Prog. Theor. Phys. 30, 204.

[11] Boldt, E. and Serlemitsos, P.: 1969, paper presented at 128th meeting of A.A.S., Austin, Texas (December).

[12] Gould, R. J. and Burbidge, G. R.: 1963, Astrophys J. 138, 969.

[13] Seward, F., Chodil, G., Mark, H., Swift, C., and Toor, A.: 1967, Astrophys. J. $150,845$.

[14] Heitler, W.: 1954, The Quantum Theory of Radiation, Clarendon Press, Oxford, Sec. 21. 
[15] Bethe, H. A. and Salpeter, E. E.: 1957, Quantum Mechanics of One-And-Two Electron Atoms, Springer-Verlag, Berlin, Sec. IVb.

[16] Fink, R. W., Jopson, R. C., Mark, Hans, and Swift, ,C. D. 1966, Rev. Mod. Phys. 38, 526.

[17] Allen, C. W.: 1963, Astrophysical Quantities, The Athlone Press, London.

[18] Bell, K. L. and Kingston, A. E.: 1967, Monthly Notices Roy. Astron. Soc. 136, 241.

[19] Gorenstein, P., Kellogg, E. M., and Gursky, H.: 1969, Astrophys. J., 156, 315

[20] Merzbacher, E., and Lewis, H. W.: 1958, Handbuch der Physik, Vol. 34, 166.

[21] Bates, D. R. and Griffing, G.: 1953, Proc. Phys. Soc. A66, 961.

[22] Comstock, G. M., Fan, C. Y., and Simpson, J. A.: 1969, Astrophys. J. 155, 609.

[23] Bethe, H. A. and Salpeter, E. E.: 1957, Quantum Mechanics of One-and-Two Electron Atoms Springer-Verlag, Berlin, Sec. IVa.

[24] Bates, D. R. and Dalgarno A.: 1953 Proc. Phys. Soc. A66 972.

[25] Hiskes, J. R.: 1965, Phys. Rev. 137A, 361.

[26] Bearden, J. A.: 1967, Rev. Mod. Phys. 39, 78.

[27] Bearden, J. A.: 1967, Rev. Mod. Phys. 39, 125. 